\title{
Maryland Bridge : A Treatment Option in Children
}

\author{
Priyanka Arghode*, Prashant Babaji and Shashibhushan KK \\ Department of Pedodontics and Preventive Dentistry, Sharavathi Dental College and Hospital, India
}

*Corresponding author: Priyanka Arghode, Department of Pedodontics and Preventive Dentistry, Sharavathi Dental College and Hospital, India

\begin{abstract}
The most common traumatic injury encountered in a pediatric dental set up is avulsion. Loss of permanent anterior teeth at a young age can have an impact on the psychological development of the child. Reimplantation if not possible, the anterior tooth can be restored with either removable or fixed prosthesis depending on the completion of the growth. To overcome the limitations of removable prosthesis, temporary fixed prosthesis can be an option in restoring the esthetics and functions when growth is not completed. Maryland bridge is an ideal option as a fixed temporary prosthesis in such patients.
\end{abstract}

Keywords: Avulsion; maryland bridge; re-implantation

\section{Introduction}

Over the last few decades dentistry has taken its turn towards more conservative and preventive treatment modalities. This has been possible not only because of the improved and advanced materials and techniques but also because of the understanding to preserve the natural tooth as far as possible. Reasons for tooth loss could be congenital absence, caries or trauma. Of these, traumatic injuries resulting in avulsion of the tooth is one of the common causes for tooth loss in the anterior region of the oral cavity. Trauma to the anterior teeth is common in childhood, one study reported that out of 2,100 children (aged 8-14 years) surveyed for teeth fractured due to trauma, $60.74 \%$ were aged between 11 and 14 with $13.8 \%$ cases involved incisors [1]. This case report presents a case of replacement of missing anterior teeth because of avulsion with direct fiber reinforced resin composite.

\section{Case Report}

A 13-year-old boy reported to the Department of Pedodontics and Preventive dentistry with a chief complaint of avulsed tooth in the maxillary front region of the jaw for three weeks. Patient had a fall while riding bicycle. The patient was healthy without any significant medical and dental history. On extra-oral examination no abnormalities were found. On intra-oral examination soft tissues were normal avulsed socket was all healed. Patient had no bruises or scars on his body after the fall. Maxillary right lateral incisor had avulsed was seen with respect to maxillary right lateral incisor (Figures 1\&2). Patient had reported to our department after three weeks with his natural avulsed tooth. The avulsed tooth was not stored in any media by the patient and was not in a good condition to be used as pontic as it had become dehydrated and had lost its color from the normal one. As the patient growth was not completed implant was not an option. Patient was very concerned about his esthetics and wanted immediate replacement. An option of conservative and conventional fiber reinforced composite resin bonded pontic was selected for the prosthetic rehabilitation. A composite buildup of maxillary right lateral incisor was done on a diagnostic cast. On the diagnostic cast the length of the FRC fiber (everstick, GC company) was pre-measured from the half the maxillary right canine to the half of the maxillary right central incisor. The fiber was pre-measured in such a way that it followed the contour of the ridge.

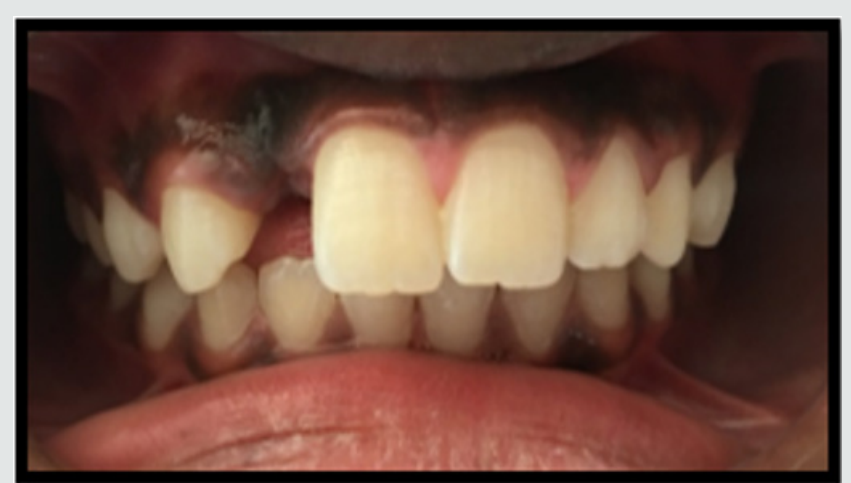

Figure 1: Intraoral view showing missing maxillary right lateral incisor. 


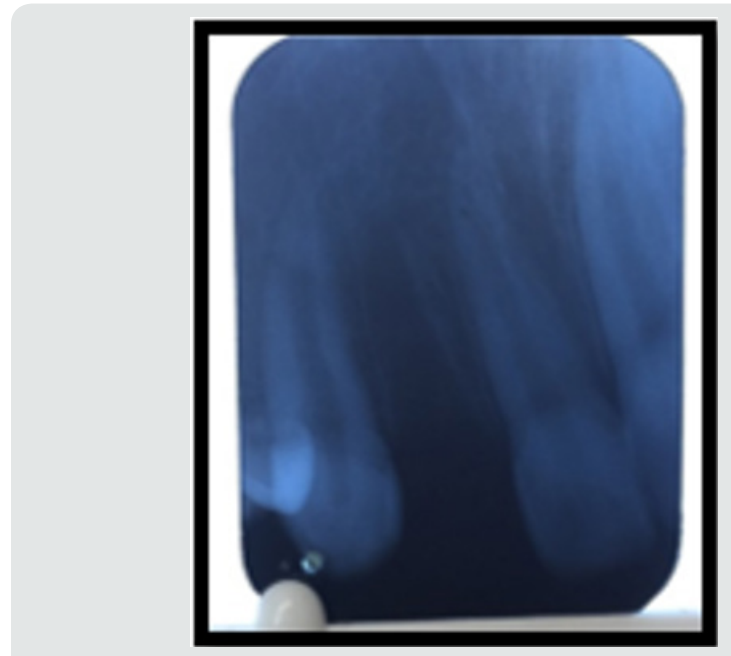

Figure 2: Radiographic view.

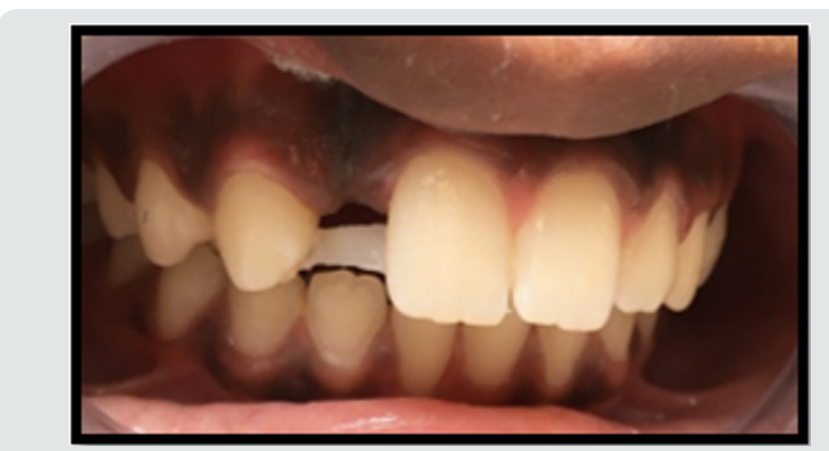

Figure 3: Ever stick fiber placed on palatal Grooves.

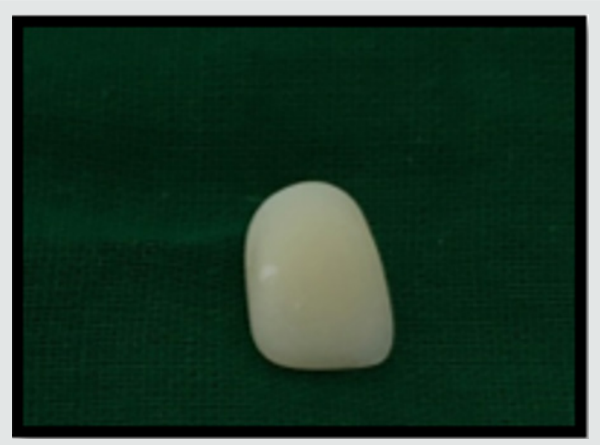

Figure 4: Composite build-up pontic.

The palatal surface of the maxillary right canine and the right central incisor was roughened for better adaptability of the fiber for good retention. The surfaces were roughened using coarse flame shaped bur. Then the roughened surfaces of the abutment teeth was etched and bonded and then the fiber was placed starting from the canine to the central incisor and it was well adapted on the surface with a plastic carrying instrument. Then the fiber was bonded using composite in such a way that the fiber was at the center of the bonded tooth and was not in occlusion (Figure 3). Then a groove was made palatally on the composite build up pontic so that the fiber inserts well in the pontic for better retention (Figure 4). The pontic was bonded with composite keeping the arch form and function in harmony (Figures 5\&6). The roughened surfaces were smoothened and polished with finishing and polishing kit (Enhance
Composite Finishing and Polishing System Dentsply Caulk Dentsply International Inc, Milford, DE). The patient was followed for 6 months and had no complaints with the prosthesis.

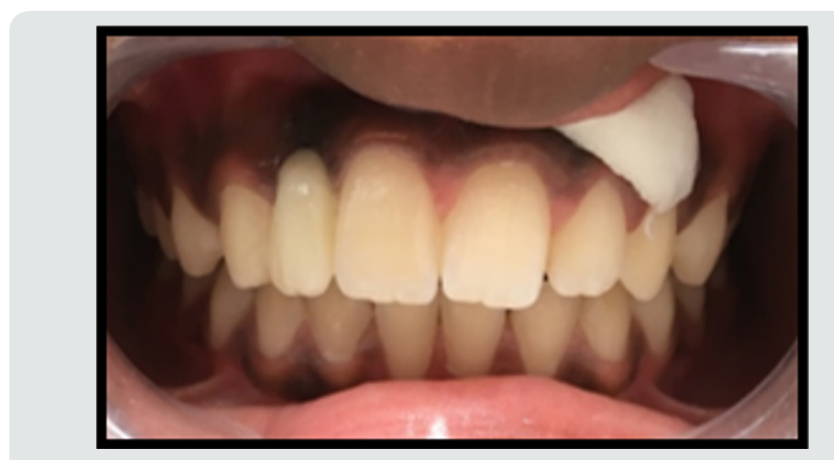

Figure 5: Fiber reinforced bridge replacing missing tooth.

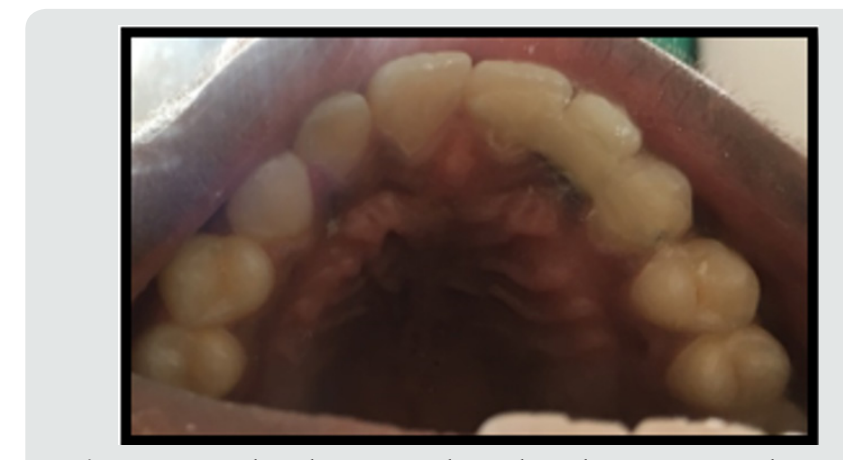

Figure 6: Occlusal view with replaced missing tooth.

\section{Discussion}

The patients with lost anterior teeth require immediate attention for the restoration of the aesthetics and function. The various treatment options available for restoring missing anterior tooth after avulsion are reimplantation, removable partial denture or fixed prosthesis depending on the case. In the present case the patient reported with the avulsed tooth after 3 weeks so reimplantation of the tooth was not a treatment option. Removable partial dentures are the most easily fabricated and cheapest options available, but they are often unacceptable to the patient because they are bulky, uncomfortable and not very esthetically pleasing [2]. Replacement of missing teeth with conventional fixed partial denture can involve tooth preparation of abutment teeth which can cause hypersensitivity, pulpal injury [3]. Fiber reinforced bridge was opted in this case, in order to provide a single visit, cost effective and a minimally invasive fixed solution to the patient. Clinical studies have shown a substantial clinical performance of the FRC FPDs, with an overall survival rate of $75 \%$ after about 5 years, which are higher than that of the FPDs with metal frameworks [4]. Hence high pulp horns of abutment teeth, expected transition in the position of the gingiva and age of the patient were factors that precluded the use of conventional fixed prostheses in this case. The resin material used in this study was GC Ever stick as a bridge for replacement of missing tooth. Accurate bonding between the fibers and composite is the key factor for a successful treatment. The resin fiber used has a unique, interpenetrating polymer network 
structure (IPN) which leads to superior bonding enabling reliable surface retained applications and perfect handling properties. The use of FRC bridges also allows utilization of a patient's natural crown as a pontic if the tooth or tooth crown is still intact [5]. In our case the lost tooth was fractured and was not in good condition to be used as a pontic, so the composite build up tooth was used (Figure 4). A study was done involving 358 patients which concluded that the patient's acceptance was better with resin bonded fixed partial dentures [6]. Maryland bridge is a minimal invasive procedure with less harm to the abutment tooth, minimum chair side time and less cost. It is well accepted by young patients and can be used as a treatment option for avulsion cases.

\section{References}

1. Gupta K, Tandon S, Prabhu D (2002) Traumatic injuries to the incisors in children of South Kanara district: A prevalence study. J Indian Soc Pedod Prev Dent 20(3): 107-113.
2. Holt LR, Drake B (2008) The Procera Maryland Bridge: A case report. J Esthet Restor Dent 20(3): 165-171.

3. Sabita M Ram, Deshpande P Rubina (2010) The zirconia resin bonded prosthesis: A case report. Dental practice 9(2): 12-14.

4. Vallittu PK (2004) Survival rates of resin-bonded, glass fiber-reinforced composite fixed partial dentures with a mean follow-up of 42 months: a pilot study. J Prosthet Dent 91(3): 241-46.

5. Belli S, Ozer F (2000) A simple method for single anterior tooth replacement. J Adhes Dent 2(1): 67-70.

6. Creugers NH, De Kanter RJ (2000) Patients' satisfaction in two long term clinical studies on resin-bonded bridges. J Oral Rehabil 27(7): 602-607.
(C) (P) This work is licensed under Creative

To Submit Your Article Click Here: Submit Article

DOI: $10.32474 /$ IPDOAJ.2020.03.000172

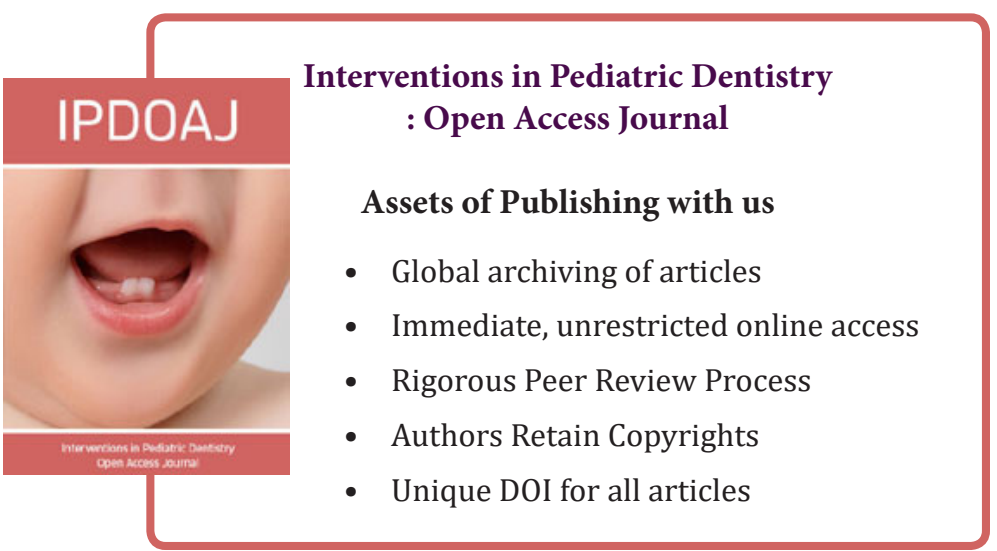

